Bài báo khoa học

\title{
Nghiên cứu đặc điểm và sự tích lũy vi nhựa trong than bùn tại xã Thuận Nghĩa Hòa, huyện Thạnh Hóa, tỉnh Long An
}

\author{
Nguyễn Minh Kỳ 1,2*, Nguyễn Tri Quang Hưng², Nguyễn Công Mạnh ${ }^{2}$, Bạch Quang \\ Dũng ${ }^{3}$ \\ ${ }^{1}$ Khoa Môi trường và Tài nguyên, Trường Đại học Nông Lâm TP. Hồ Chí Minh; \\ quanghungmt@hcmuaf.edu.vn \\ ${ }^{2}$ Bộ môn Môi trường và Tài nguyên, Trường Đại học Nông Lâm TP. Hồ Chí Minh - Cơ \\ sở Gia Lai; nmky@hcmuaf.edu.vn \\ ${ }^{3}$ Phòng 1703, Chung cư 25, Tân Mai, Phường Tân Mai, Q. Hoàng Mai, TP. Hà Nội; \\ dungmmu05@gmail.com
}

*Tác giả liên hệ: nmky@hcmuaf.edu.vn; Tel.: +84-916121204

Ban Biên tập nhận bài: 08/11/2021; Ngày phản biện xong: 31/12/2021; Ngày đăng bài: $25 / 2 / 2022$

Tóm tắt: Mục đích nghiên cứu được thực hiện nhằm đánh giá đặc điểm và sự tích lũy vi nhựa trong trầm tích than bùn, trường hợp nghiên cứu điển hình ở tỉnh Long An. Nghiên cứu tiến hành khảo sát thực địa và chọn lấy mẫu đại diện tại 10 vị trí khác nhau trong khu vực có các lớp than bùn thuộc địa bàn xã Thuận Nghĩa Hòa, huyện Thạnh Hóa, Long An. Các mẫu được xử lý nhằm xác định hàm lượng, kích thước hạt, màu sắc, hình dạng và định danh chủng loại vi nhựa. Mức độ nhiễm bẩn vi nhựa cho thấy hàm lượng trong than bùn tại Long An có sự dao động 0-360,0 hạt/kg. Vi nhựa có kích thước tương đối lớn, hình dạng chủ yếu là dạng mảnh $(69,6 \%)$, bọt $(17,4 \%)$, sợi $(8,7 \%)$ và dạng film $(4,3 \%)$, chúng thường có màu trắng, nước biển, xanh. Về thành phần vi nhựa được phát hiện gồm các loại như polypropylene $(\mathrm{PP})$, polyethylene $(\mathrm{PE})$, polyvinyl chloride $(\mathrm{PVC})$ và các dạng sợi polyester; trong đó, nhựa $\mathrm{PVC}$ chiếm chủ yếu với tỷ lệ $70,6 \%$. Kết quả nghiên cứu cũng cho thấy sự phân bố vi nhựa theo không gian và chỉ thị dấu hiệu tác động của con người lên hiện trạng than bùn. Do đó, về lâu dài cần có những giải pháp thích hợp nhằm quản lý, bảo vệ nguồn tài nguyên than bùn một cách hữu hiệu trước các áp lực phát triển kinh tế-xã hội.

Từ khóa: Vi nhựa; Nhiễm bẩn; PVC; Than bùn; Polypropylene; Tích lũy.

\section{1. Đặt vấn đề}

Ngày nay, vấn đề nhiễm bẩn rác thải nhựa là tiêu điểm nổi bật trong các thách thức liên quan đến sinh thái môi trường. Theo các thống kê trên toàn thế giới cho thấy tốc độ sản xuất nhựa không ngừng gia tăng từ 1,7 triệu tấn năm 1950 lên 335 triệu tấn năm 2017 [1]. Vi nhựa (microplastic) là chất ô nhiễm mới nổi, có khả năng đe dọa nghiêm trọng đến hệ sinh thái và chúng được định nghĩa là các mảnh nhựa có kích thước nhỏ hơn $5 \mathrm{~mm}$ [2]. Vi nhựa được phân loại theo 2 hình thức, gồm các hạt vi nhựa sơ cấp và thứ cấp. Vi nhựa sau khi xâm nhập vào cơ thể và được xem như là chất có nguy cơ gây độc cho sức khỏe con người. Sự nhiễm bẩn vi nhựa đã được tìm thấy ở khắp nơi, chúng tồn tại ở các điều kiện môi trường khác nhau từ đất, nước, không khí cho tới các nguồn thực phẩm [3-4]. Kết quả nghiên cứu [5] cho thấy hàm lượng vi nhựa trong trầm tích tại Pháp là 67 hạt $/ \mathrm{kg}$. Tương tự, mức độ nhiễm bẩn vi nhựa trong trầm tích ở Tây Ban Nha chủ yếu gồm các dạng mảnh, sợi 
với hàm lượng xác định là 100-900 hạt/kg [6]. Không những vậy, tỷ lệ tái chế nhựa thấp ở các quốc gia đang phát triển sẽ càng làm gia tăng nguy cơ và sự lo lắng về mặt môi trường, sức khỏe [7]. Trong khi, Việt Nam là một trong những đất nước có mức độ tiêu thụ nhựa cao nhất khu vực Đông Nam Á [8] và cũng như thuộc trong số 20 quốc gia có sự chậm trễ, thiếu hiệu quả trong việc quản lý rác thải nhựa [9]. Quá trình thiếu sự quản lý chặt chẽ rác thải nhựa sẽ gây ra những vấn đề khủng hoảng về mặt môi trường liên quan đến vấn nạn nhiễm bẩn vi nhựa. Có thể thấy, sự cần thiết đánh giá hiện trạng nhiễm bẩn nhựa trong tiến trình quản lý và kiểm soát để bảo vệ môi trường. Chính vì lẽ đó, đã có một số nhóm nghiên cứu tiến hành lấy mẫu, phân tích và đánh giá hiện trạng nhiễm bẩn vi nhựa ở Việt Nam [810]. Thực tế cho thấy số lượng báo cáo về nhiễm bẩn nhựa vẫn còn nhiều hạn chế. Các nghiên cứu trước đây chủ yếu tập trung đánh giá sự nhiễm bẩn vi nhựa ở môi trường nước [11], trầm tích [10-12], động vật hai mảnh vỏ [13], cát biển [14], hải sản [15] hay trong môi trường không khí [16]. Hiện trạng ô nhiễm rác thải nhựa nói chung, vi nhựa nói riêng đã và đang là vấn đề nổi cộm. Tuy nhiên, thực tế hiện nay chưa có công bố nào thực hiện trên đối tượng than bùn.

Mặc dù than bùn (peatland) chỉ chiếm $3 \%$ tổng diện tích các lục địa $\left(5\right.$ triệu $\left.\mathrm{km}^{2}\right)$ nhưng lại có ý nghĩa quan trọng đối với các giá trị của hệ sinh thái toàn cầu [17]. Sự tích lũy vi nhựa trong tầng trầm tích than bùn sẽ có thể gây ảnh hưởng đến đời sống các loài thủy sinh và thông qua các chuỗi thức ăn tác động đến sức khỏe con người [18]. Do lượng vi nhựa trong than bùn được tiêu thụ bởi một số loài thủy sinh như cá, tôm.. hay như sự tích lũy trong canh tác nông nghiệp. Từ đó, sẽ gây ra những ảnh hưởng tiềm tàng thông qua chuỗi thức ăn, hậu quả là vấn đề sức khỏe cho con người. Mặt khác, Long An là tỉnh tiếp giáp với Thành phố Hồ Chí Minh, Tây Ninh ở phía Đông, giáp với Campuchia ở phía Bắc, tỉnh Đồng Tháp ở phía Tây và Tiền Giang về phía Nam. Tỉnh Long An có vị trí địa lý đặc biệt quan trọng, nằm ở vùng đồng bằng sông Cửu Long và thuộc vùng phát triển kinh tế trọng điểm phía Nam. Đây là cấu phần không thể thiếu của vùng kinh tế động lực trong chiến lược phát triển kinh tế-xã hội đồng bằng sông Cửu Long. Hơn nữa, Long An có địa hình bằng phẳng, có xu thế thấp dần từ phía Bắc-Đông Bắc xuống Nam-Tây Nam [19]. Địa hình bị chia cắt bởi hai dòng sông lớn (Vàm Cỏ Đông và Vàm Cỏ Tây), và với hệ thống kênh rạch chằng chịt, phần lớn diện tích đất của tỉnh Long $A n$ được xếp vào vùng đất ngập nước. Do đó, đã hình thành nên những lớp than bùn đầm lầy lâu đời. Các mỏ than bùn đầm lầy ở đây có quy mô nhỏ và được phân bố rải rác nhưng có ý nghĩa trong trọng về mặt môi trường cũng như sinh kế người dân. Theo kết quả điều tra của dự án quản lý bền vững than bùn khu vực Đông Nam Á, than bùn ở Long An phát hiện ở các huyện như Thạnh Hóa, Tân Thạnh và Đức Huệ với tổng diện tích khoảng 72 ha [20]. Than bùn là nguồn nguyên liệu khá tốt để chế biến ra nhiều loại sản phẩm có giá trị kinh tế cao [21-22]. Các kết quả phân tích và đánh giá chất lượng cho thấy than bùn ở Long $A n$ dày $0,5-5,0 \mathrm{~m}$, độ tro thấp, lượng khoáng cao, mùn cao, có thể sử dụng làm chất đốt và phân bón. Tuy vậy, trong những năm gần đây với tốc độ tăng trưởng kinh tế cao ở Long An đã tạo nên áp lực về mặt môi trường. Các hoạt động sản xuất, kinh doanh trên địa bàn đóng góp vào sự nhiễm bẩn nói chung và rác thải nhựa nói riêng. Do đó, rất cần những nghiên cứu đánh giá đặc điểm và sự tích lũy thành phần vi nhựa để có cơ sở đề ra các giải pháp quản lý thích hợp. Nghiên cứu này được thực hiện nhằm xem xét mức độ nhiễm bẩn thông qua việc lấy mẫu, phân tích và đánh giá đặc điểm và sự tích lũy vi nhựa trong than bùn, trường hợp nghiên cứu điển hình ở xã Thuận Nghĩa Hòa, huyện Thạnh Hóa, tỉnh Long An.

\section{Phương pháp nghiên cứu}

\subsection{Khảo sát thục địa và lấy mẫu}

Nghiên cứu tiến hành khảo sát thực địa và chọn mẫu đại diện ở 10 vị trí khác nhau trong khu vực chứa than bùn thuộc địa bàn xã Thuận Nghĩa Hòa, Thạnh Hóa, Long An (Hình 1). Liên quan đến vị trí các điểm lấy mẫu được mô tả trong Bảng 1. 
Bảng 1. Vị trí các điểm lấy mẫu.

\begin{tabular}{|c|c|c|c|}
\hline \multirow{2}{*}{ TT } & \multirow{2}{*}{ Ký hiệu } & \multicolumn{2}{|c|}{ Tọa độ } \\
\hline & & Vĩ độ & Kinh độ \\
\hline 1 & TH1 & $10^{\circ} 39^{\prime} 53.1 ’ \mathrm{~N}$ & $106^{\circ} 11^{\prime} 06.0^{\prime \prime} \mathrm{E}$ \\
\hline 2 & TH2 & $10^{\circ} 39^{\prime} 56.1 ’ \mathrm{~N}$ & $106^{\circ} 11^{\prime} 10.6^{\prime \prime} \mathrm{E}$ \\
\hline 3 & TH3 & $10^{\circ} 39^{\prime} 59.8^{\prime \prime} \mathrm{N}$ & $106^{\circ} 11^{\prime} 05.0^{\prime \prime} \mathrm{E}$ \\
\hline 4 & TH4 & $10^{\circ} 40^{\prime} 00.9 " \mathrm{~N}$ & $106^{\circ} 11 ' 10.5$ 'E \\
\hline 5 & TH5 & $10^{\circ} 40^{\prime} 04.1 ” \mathrm{~N}$ & $106^{\circ} 11^{\prime} 18.4^{\prime \prime} \mathrm{E}$ \\
\hline 6 & TH6 & $10^{\circ} 40^{\prime} 16.9$ ' $\mathrm{N}$ & $106^{\circ} 11^{\prime} 24.5^{\prime \prime} \mathrm{E}$ \\
\hline 7 & TH7 & $10^{\circ} 40^{\prime} 20.4 ” \mathrm{~N}$ & $106^{\circ} 11^{\prime} 03.3$ 'Е \\
\hline 8 & TH8 & $10^{\circ} 40^{\prime} 23.2^{\prime \prime} \mathrm{N}$ & $106^{\circ} 11^{\prime} 13.1^{\prime \prime} \mathrm{E}$ \\
\hline 9 & TH9 & $10^{\circ} 40^{\prime} 10.8 ’ \mathrm{~N}$ & $106^{\circ} 11^{\prime} 21.4^{\prime \prime} \mathrm{E}$ \\
\hline 10 & TH10 & $10^{\circ} 40^{\prime} 24.0 ’ \mathrm{~N}$ & $106^{\circ} 11^{\prime} 27.5^{\prime \prime} \mathrm{E}$ \\
\hline
\end{tabular}

Phương pháp lấy mẫu dựa theo nguyên tắc lấy mẫu định hướng. Căn cứ quá trình khảo sát thực địa nghiên cứu lựa chọn các vị trí tập trung than bùn và đại diện trong khu vực. Mẫu trầm tích than bùn được lấy bằng gầu lấy mẫu, diện tích mẫu là $500 \mathrm{~cm}^{2}$. Các mẫu được lấy ở độ sâu so với tầng mặt với từ $5-15 \mathrm{~cm}$. Để đảm bảo tính đại diện, than bùn từ gầu được thả ra khay và tiến hành trộn đều trước khi cho vào các túi giấy nhôm (aluminum foil bag) chuyên dụng. Khối lượng mỗi mẫu được lấy tương đương 500 gram. Mẫu sau lấy được bảo quản lưu trữ và vận chuyển về phòng thí nghiệm để phân tích xác định đặc điểm, định danh thành phần vi nhựa $[12,23]$.
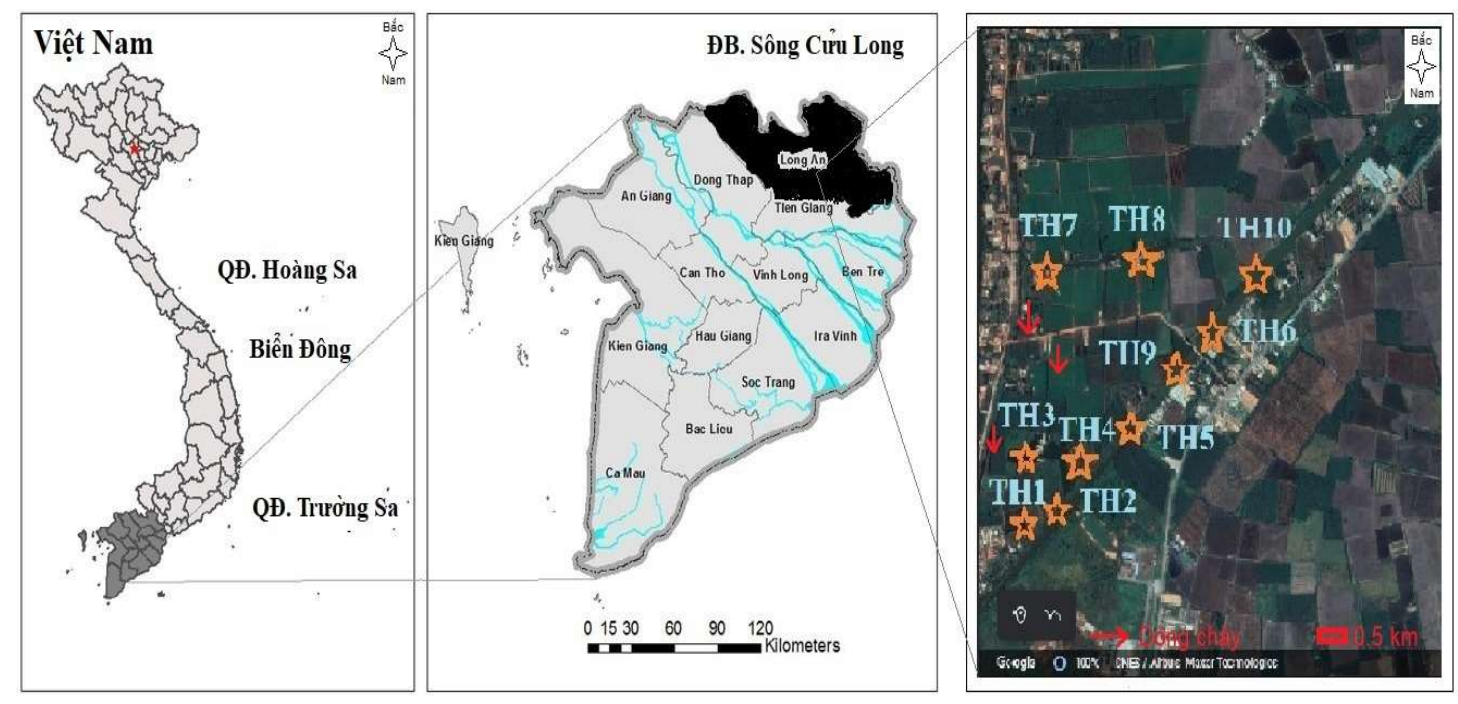

Hình 1. Sơ đồ vị trí các điểm lấy mẫu tại xã Thuận Nghĩa Hòa, huyện Thạnh Hóa.

\subsection{Xử lý và phân tích mẫu}

Các mẫu được xử lý nhằm xác định hàm lượng (hạt $/ \mathrm{kg})$, kích thước hạt $(\mathrm{mm})$, màu sắc (trắng, xanh, đỏ,..), hình dạng (hình cầu, sợi, mảnh,..) và loại nhựa (PE, PP, $\mathrm{PVC}, .$.$) . Trong$ đó, kích thước, màu sắc và hình dạng được xác định bởi thiết bị kính hiển vi soi nổi, độ phóng đại 10x (Optika B-292PLi). Phương pháp hấp thụ bức xạ hồng ngoại FT-IR (Fourrier Transformation InfraRed) được sử dụng để xác định loại nhựa trong các mẫu nghiên cứu. Quá trình xử lý và phân tích mẫu được mô tả như Hình 2 [11, 12, 23].

\subsection{Phân tích thống kê}

Các số liệu thu thập được tính toán giá trị trung bình $(\overline{\mathrm{x}})$ và độ lệch chuẩn $\left(\mathrm{S}_{\mathrm{x}}\right)$ theo công thức: $\mathrm{S}=\sqrt{ }\left(\sum\left(\left(\mathrm{x}_{\mathrm{i}}-\mathrm{x}\right)^{2} / \mathrm{n}-1\right)\right.$. Trong đó, $\mathrm{x}_{\mathrm{i}}$ là giá trị trên quan sát thứ $\mathrm{i}$ của mẫu nghiên cứu và $\mathrm{n}$ là kích thước mẫu. Thủ tục phân tích thống kê thực hiện bằng phần mềm SPSS 16.0 for Windows. 


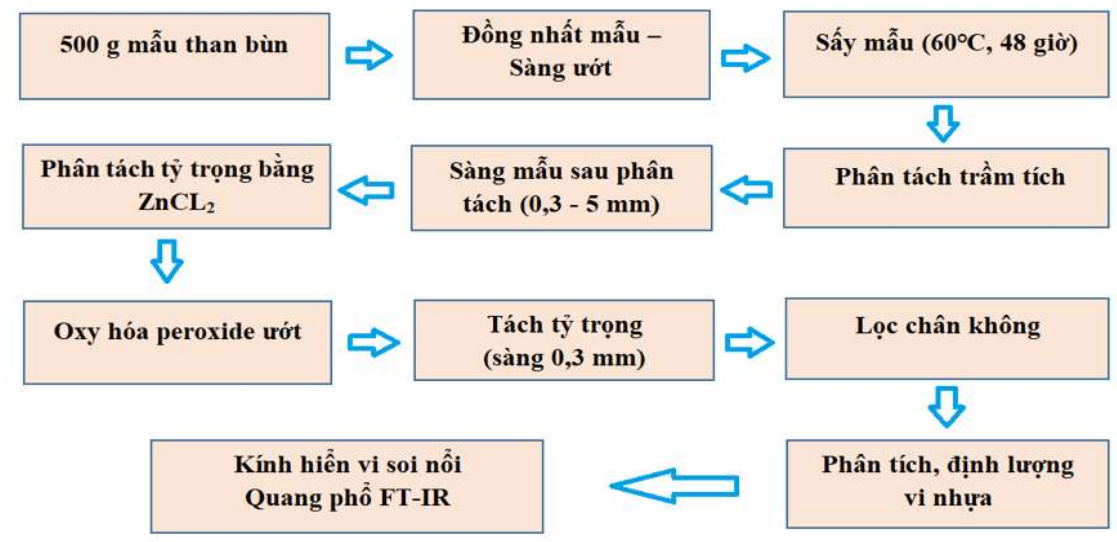

Hình 2. Quy trình xử lý và phân tích vi nhựa.

\section{Kết quả nghiên cứu và thảo luận}

\subsection{Sự tích lũy vi nhựa trong thành phần than bùn}

Kết quả nghiên cứu cho thấy sự tích lũy hàm lượng vi nhựa tại các địa điểm lấy mẫu than bùn ở Thạnh Hóa chủ yếu tập trung ở vị trí TH7 (Hình 3). Trong đó, số lượng vi nhựa chiếm lớn nhất với sự có mặt các loại kích thước khác nhau $(0,3-5,0 \mathrm{~mm})$ đạt giá trị trung bình $360,0 \pm 4,7$ hạt $/ \mathrm{kg}$. Các kết quả tại vị trí lấy mẫu $\mathrm{TH} 1$ và $\mathrm{TH} 9$ lần lượt với hàm lượng $60,0 \pm 2,5$ và $50,0 \pm 1,3$ hạt $/ \mathrm{kg}$. Như vậy, số lượng vi nhựa trong than bùn ở Thạnh Hóa lần lượt thứ tự: TH7 > TH1 > TH9 > TH2, TH3, TH4, TH5, TH6 > TH8, TH10. Quá trình thực địa cho thấy sự khác biệt ở các vị trí lấy mẫu nêu trên là do sự tiếp giáp dân cư đông đúc và các hoạt động đi kèm của con người. Nhìn chung, mức độ nhiễm bẩn vi nhựa tại khu vực nghiên cứu còn thấp tuy nhiên phần nào cảnh báo đã có sự ảnh hưởng từ các nguồn xả thải, tác động và gây ra sự xuất hiện hàm lượng vi nhựa trong thành phần than bùn. Thông thường, sự nhiễm bẩn vi nhựa có thể do từ các nguồn xả thải nước thải (công nghiệp và sinh hoạt), các hoạt động nông nghiệp, sự chảy tràn bề mặt và ô nhiễm không khí [24-25]. Đối với hàm lượng vi nhựa xuất hiện với sự tích lũy cao ở địa điểm lấy mẫu TH7 có thể giải thích bởi khu vực tiếp cận với nguồn xả thải từ hoạt động nông nghiệp cũng như sự ảnh hưởng của lượng nước chảy tràn và một phần gián tiếp do hoạt động dân sinh xung quanh. Trong các hoạt động nông nghiệp, việc sử dụng các lớp phủ nhựa là nguyên nhân phát thải lượng lớn thành phần vi nhựa như polyvinyl chloride (PVC), polypropylene (PP), polyethylene $(\mathrm{PE})$, v.v.. Hoạt động canh tác sử dụng phân bón hữu cơ từ compost cũng là nguyên đóng góp vào sự nhiễm bẩn vi nhựa trong môi trường đất [26]. Thông qua các hoạt động thường nhật, vi nhựa còn được phát sinh từ thói quen sử dụng chai nhựa, bao bì, sợi tổng hợp,.. trong các hộ dân cũng như các sản phẩm chăm sóc cá nhân như dầu gội, sữa rửa mặt, son môi,... Có thể thấy rằng, quá trình nhiễm bẩn rác thải nhựa và hậu quả sâu xa là sự nhiễm bẩn vi nhựa vào môi trường đặc biệt các vùng sinh thái quan trọng như đất nông nghiệp, khu vực đầm lầy, hệ sinh thái ven sông là vấn đề môi trường cần được quan tâm giải quyết [26-29]. Do đó về lâu dài cần có những cảnh báo sớm và giải pháp quản lý thích hợp nhằm hạn chế sự ô nhiễm rác thải nhựa nhằm hạn chế tác động đến nguồn tài nguyên than bùn.

Để xem xét sự tích lũy và phân bố nhiễm bẩn vi nhựa tại các khu vực ở Việt Nam và trên thế giới, Bảng 2 tổng hợp so sánh kết quả các hàm lượng của chúng trong môi trường nước, trầm tích. Từ mức độ nhiễm bẩn vi nhựa cho thấy, hàm lượng tại Long An có dao động trong khoảng giá trị 0-360,0 hạt $/ \mathrm{kg}$, thấp hơn nhiều so với môi trường nước mặt ở sông Sài Gòn và kênh rạch phụ cận (10-519000 hạt $\left./ \mathrm{m}^{3}\right)$ hay như trong trầm tích bờ biển Đà Nẵng $(9238 \pm 2097$ hạt $/ \mathrm{kg})$ và trầm tích bãi triều ở Thanh Hóa (2921-5365 hạt $/ \mathrm{kg})$. Tuy nhiên, giữa chúng lại có sự khá tương đồng tại các mẫu trầm tích sông Sài Gòn-Đồng Nai (38-498 hạt $/ \mathrm{kg})$. Bảng 2 còn thể hiện so sánh hàm lượng vi nhựa ở trầm tích một số quốc 
gia ở Châu Âu. Nghiên cứu thực hiện tại vịnh Biscay (Pháp) và đảo Pianosa (Ý) chỉ ra hàm lượng vi nhựa tích lũy trong trầm tích lần lượt $67 \mathrm{hạt} / \mathrm{kg}$ và $1,09 \mathrm{~g} / \mathrm{m}^{2}$. Có thể thấy, với mức độ đô thị hóa nhanh cùng với áp lực xả thải từ các nguồn công nghiệp trong hoạt động sản xuất, kinh doanh đã góp phần gia tăng nhanh chóng mức độ nhiễm bẩn vi nhựa vào môi trường [24].

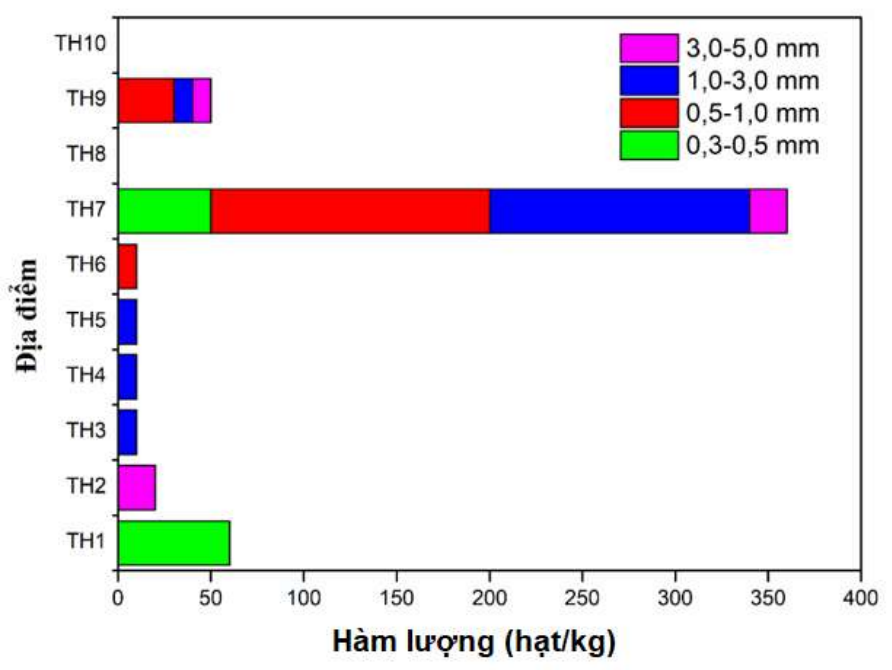

Hình 3. Mức độ tích lũy vi nhựa trong than bùn ở Thạnh Hóa.

Bảng 2. So sánh mức độ nhiễm bẩn vi nhựa trong môi trường ở Việt Nam và thế giới.

\begin{tabular}{|c|c|c|c|c|c|c|}
\hline Địa phương & Địa lý & Mô tả & Đối tượng & Hàm lượng & Hình dạng & Tham khảo \\
\hline Quảng Ninh & & - Vịnh Cửa Lục & Nước mặt & 0,35 hạt $/ \mathrm{m}^{3}$ & Sợi, mảnh & {$[30]$} \\
\hline Hà Nội & 裉 & $\begin{array}{l}\text { - Hồ Tây } \\
\text { - Sông Hồng } \\
\text { - Sông Nhuệ } \\
\text { - Sông Tô Lịch }\end{array}$ & Nước mặt & $\begin{array}{l}\text { 611,0 hạt } / \mathrm{m}^{3} \\
2,3 \text { hạt } / \mathrm{m}^{3} \\
\text { 93,7 hạt } / \mathrm{m}^{3} \\
2522 \text { hạt } / \mathrm{m}^{3}\end{array}$ & Sợi, mảnh & [30] \\
\hline Thanh Hóa & \multirow{4}{*}{ 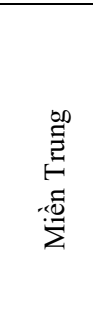 } & $\begin{array}{l}\text { - Huyện Hậu } \\
\text { Lộc }\end{array}$ & $\begin{array}{l}\text { Trầm tích } \\
\text { bãi triều }\end{array}$ & 2921-5365 hạt/kg & \multirow{2}{*}{$\begin{array}{l}\text { Mảnh, bọt, } \\
\text { sợi, film } \\
\text { Sợi, mảnh, } \\
\text { bọt, film, viên } \\
\text { nén }\end{array}$} & [12] \\
\hline \multirow[t]{2}{*}{ Đà Nẵng } & & - Đà Nẵng & $\begin{array}{l}\text { Trầm tích } \\
\text { bờ biển }\end{array}$ & $9238 \pm 2097$ hạt $/ \mathrm{kg}$ & & [10] \\
\hline & & $\begin{array}{l}\text { - Sông Hàn } \\
\text { - Hồ Công Viên }\end{array}$ & Nước mặt & $\begin{array}{l}\text { 2,7 hạt } / \mathrm{m}^{3} \\
70,0 \text { hạt } / \mathrm{m}^{3}\end{array}$ & Sợi, mảnh & [30] \\
\hline Bình Định & & - Đầm Thị Nại & Nước mặt & 3,2-24,3 hạt $/ \mathrm{m}^{3}$ & Sợi, mảnh & [30] \\
\hline Tiền Giang & \multirow{7}{*}{ 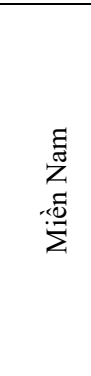 } & - Tiền Giang & Cát biển & 0-281,4 hạt/kg & Mảnh, sợi, hạt & [14] \\
\hline \multirow{2}{*}{ Vũng Tàu } & & - Sông Dinh & - Nước mặt & 28,4 hạt $/ \mathrm{m}^{3}$ & Sợi, mảnh & [30] \\
\hline & & - Vũng Tàu & - Cát biển & 0-295,0 hạt/kg & Mảnh, sợi, hạt & [14] \\
\hline \multirow{2}{*}{ Đồng Nai } & & $\begin{array}{l}\text { - Sông Sài Gòn- } \\
\text { Đồng Nai }\end{array}$ & Trầm tích & 38-498 hạt/kg & Sợi, hạt & [11] \\
\hline & & $\begin{array}{l}\text { - Hồ Trị An } \\
\text { - Sông Đồng Nai }\end{array}$ & Nước mặt & $\begin{array}{l}\text { 1,5 hạt } / \mathrm{m}^{3} \\
3,9 \text { hạt } / \mathrm{m}^{3}\end{array}$ & Sợi, mảnh & [30] \\
\hline $\begin{array}{l}\text { Tp. Hồ Chí } \\
\text { Minh }\end{array}$ & & $\begin{array}{l}\text { - Sông Sài Gòn } \\
\text { và kênh rạch }\end{array}$ & Nước mặt & $\begin{array}{l}10- \\
519000 \mathrm{hat} / \mathrm{m}^{3}\end{array}$ & Sợi, mảnh & [8] \\
\hline Long An & & $\begin{array}{l}\text { - Huyện Thạnh } \\
\text { Hóa }\end{array}$ & Than bùn & 0-360,0 hạt/kg & $\begin{array}{l}\text { Mảnh, sợi, } \\
\text { bọt, film }\end{array}$ & Nghiên cứu này \\
\hline Anh & \multirow{4}{*}{ 胥 } & - Lưu vực Tame & Trầm tích & 165,0 hạt/kg & Sợi, mảnh & [31] \\
\hline Pháp & & - Vịnh Biscay & Trầm tích & 67,0 hạt/kg & Mảnh & [5] \\
\hline \multirow{2}{*}{$\begin{array}{l}\text { Ý } \\
\text { Tây Ban } \\
\text { Nha }\end{array}$} & & \multirow{2}{*}{$\begin{array}{l}\text { - Đảo Pianosa } \\
\text { - Biển Địa Trung } \\
\text { Hải }\end{array}$} & Trầm tích & $1,09 \mathrm{~g} / \mathrm{m}^{2}$ & Mảnh & [32] \\
\hline & & & Trầm tích & 100-900 hạt/kg & Sợi, mảnh & {$[6]$} \\
\hline
\end{tabular}




\section{2. Đặc điểm vi nhưa trong than bùn}

Đặc điểm vi nhựa (kích thước, hình dạng, màu sắc, thành phần) trong than bùn ở Thạnh Hóa được thể hiện ở các Hình $4 \mathrm{a}-4 \mathrm{~d}$. Về chủng loại thành phần vi nhựa được phát hiện gồm các loại như polypropylene (PP), polyethylene (PE), polyvinyl chloride (PVC) và các dạng sợi polyester. Trong đó, nhựa PVC chiếm chủ yếu với tỷ lệ $70,6 \%$. Đối với các loại hình dạng vi nhựa gồm các thể loại chính (mảnh, sợi, bọt và film), trong đó dạng mảnh và bọt đạt tỷ lệ cao nhất (chiếm $87,0 \%$ ). Về màu sắc các loại vi nhựa khá đa dạng, gồm các màu nâu, đen, trắng, nước biển, xanh, cam, màu cát biển và màu đất. Màu sắc nước biển và trắng chiếm đa số với thành phần tương ứng $35,3 \%$ và $17,6 \%$. Liên quan đến kích thước các hạt vi nhựa được tìm thấy trong trầm tích than bùn tồn tại các khoảng giá trị $0,3-0,5$ $\mathrm{mm} ; 0,5-1,0 \mathrm{~mm} ; 1,0-3,0 \mathrm{~mm}$; và $3,0-5,0 \mathrm{~mm}$. Các hạt vi nhựa có kích thước $0,5-3,0 \mathrm{~mm}$ chiếm đa số $(74,6 \%)$, cụ thể bao gồm các hạt vi nhựa có kích thước 1,0-3,0 mm đạt mức cao nhất, với tỷ lệ 40,0\%. Ngoài vi nhựa có kích thước tương đối lớn, hình dạng chủ yếu là hình dạng mảnh $(69,6 \%)$, bọt $(17,4 \%)$, sợi $(8,7 \%)$ và dạng film $(4,3 \%)$, chúng thường có màu trắng, nước biển, xanh (Hình 5 ). Như vậy, dựa vào các đặc điểm thành phần, kích thước chủ đạo các hạt vi nhựa có thể khám phá những sự hiểu biết về sự nhiễm bẩn và tích lũy vi nhựa trong mồi trường $[33,34]$. Do phần lớn hạt vi nhựa có kích thước tương đối lớn cho thấy sự tích lũy chưa lâu và việc phân rã trong mồi trường diễn ra ở mức độ thấp. Đồng thời, các thành phần nhựa tổng hợp nhân tạo như PVC chiếm tỷ lệ cao cũng có thể lý giải bởi chúng được sử dụng phổ biến trong các hoạt động đời sống.
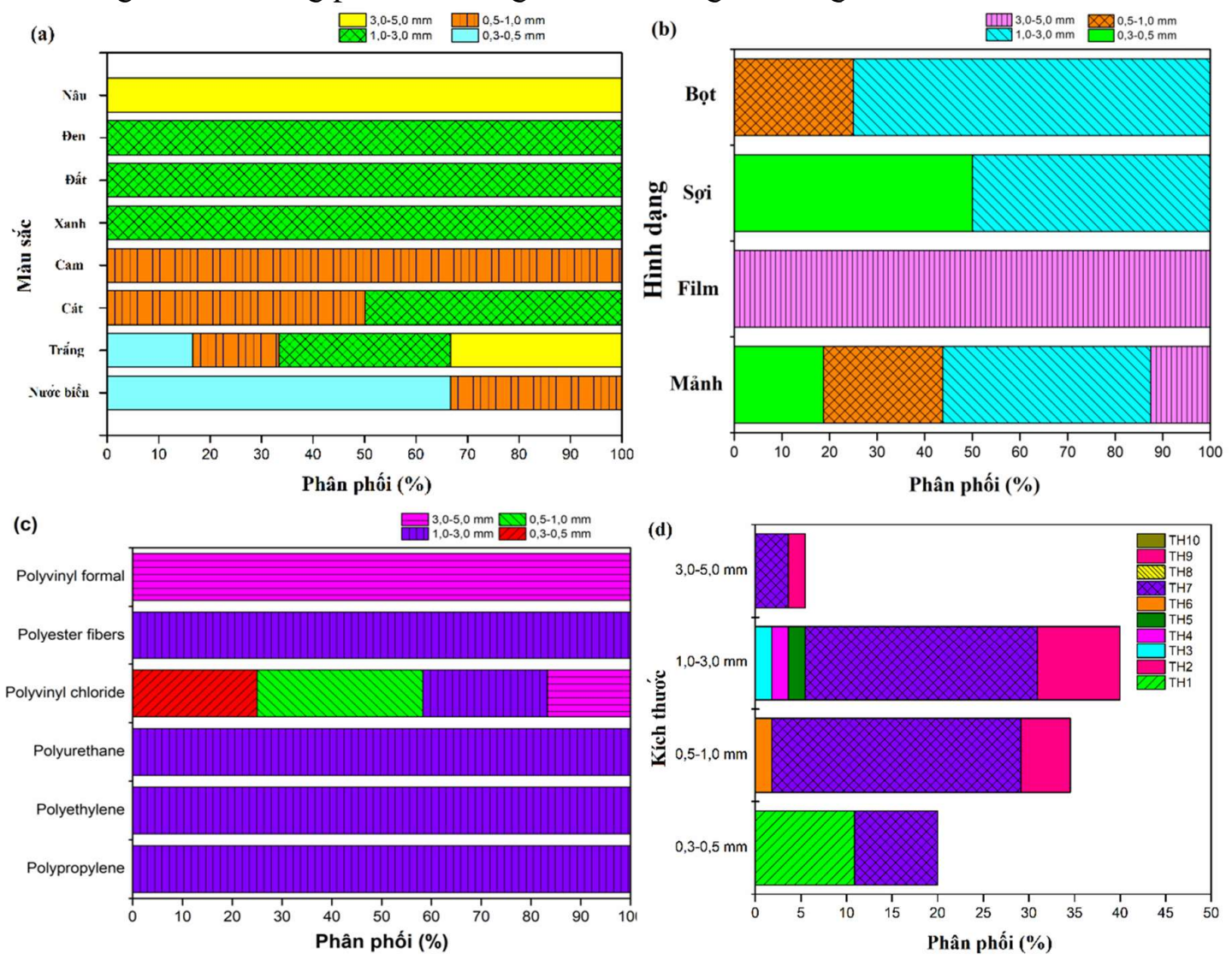

Hình 4. Đặc điểm vi nhựa (a) màu sắc, (b) hình dạng, (c) thành phần và (d) kích thước trong than bùn.

So sánh mẫu được phân tích trầm tích khu vực sông Sài Gòn-Đồng Nai có khoảng 38498 hạt $/ \mathrm{kg}$ trầm tích, mảnh nhựa có kích thước từ $0,1-0,5 \mathrm{~mm}$ là chủ yếu, trong đó nhựa thu được của PE chiếm 51,2\% [11]. Trong khi đó, kết quả phân tích trầm tích bãi triều ven biển ở Thanh Hóa cho thấy số lượng của các hạt vi nhựa dao động từ 2921 đến 5365 hạt/kg, 
với giá trị trung bình là 4123 hạt $/ \mathrm{kg}$. Theo nghiên cứu thành phần vi nhựa trong cát biển ở Tiền Giang chỉ ra rằng vi nhựa dạng mảnh chiếm ưu thế $(60,2 \%)$ trong các loại hình khác như sợi, hạt [14]. Kết quả cũng đã tiết lộ chủng loại nhựa phổ biến như PE, PP và PS được tìm thấy trong vị trí lấy mẫu. Như vậy, về cơ bản thành phần chủng loại vi nhựa giữa các nghiên cứu có sự tương đồng. Ở một nghiên cứu khác, các hạt vi nhựa có kích thước dao động trong khoảng 300-5000 $\mu \mathrm{m}$ được tìm thấy trong trầm tích bờ biển ở Đà Nẵng [10]. Hơn nữa, hàm lượng vi nhựa khám phá ở các khu vực biển Đà Nẵng có mức độ cao hơn so với các khu vực khác trên thế giới. Hầu hết chúng có dạng hình sợi, màu sắc xanh $(59,9 \%)$ và trắng $(22,9 \%)$ chiếm tỷ lệ phổ biến nhất với độ dài trung bình tương ứng $1701 \pm 1029 \mu \mathrm{m}$. Do đặc thù khác biệt về địa lý (bờ biển miền Trung so với đồng bằng sông Cửu Long) cũng như thời gian ảnh hưởng và các nguồn tác động nên hình dạng vi nhựa tìm thấy có sự khác biệt nhỏ và được thể hiện chủ yếu dưới dạng hình mảnh.
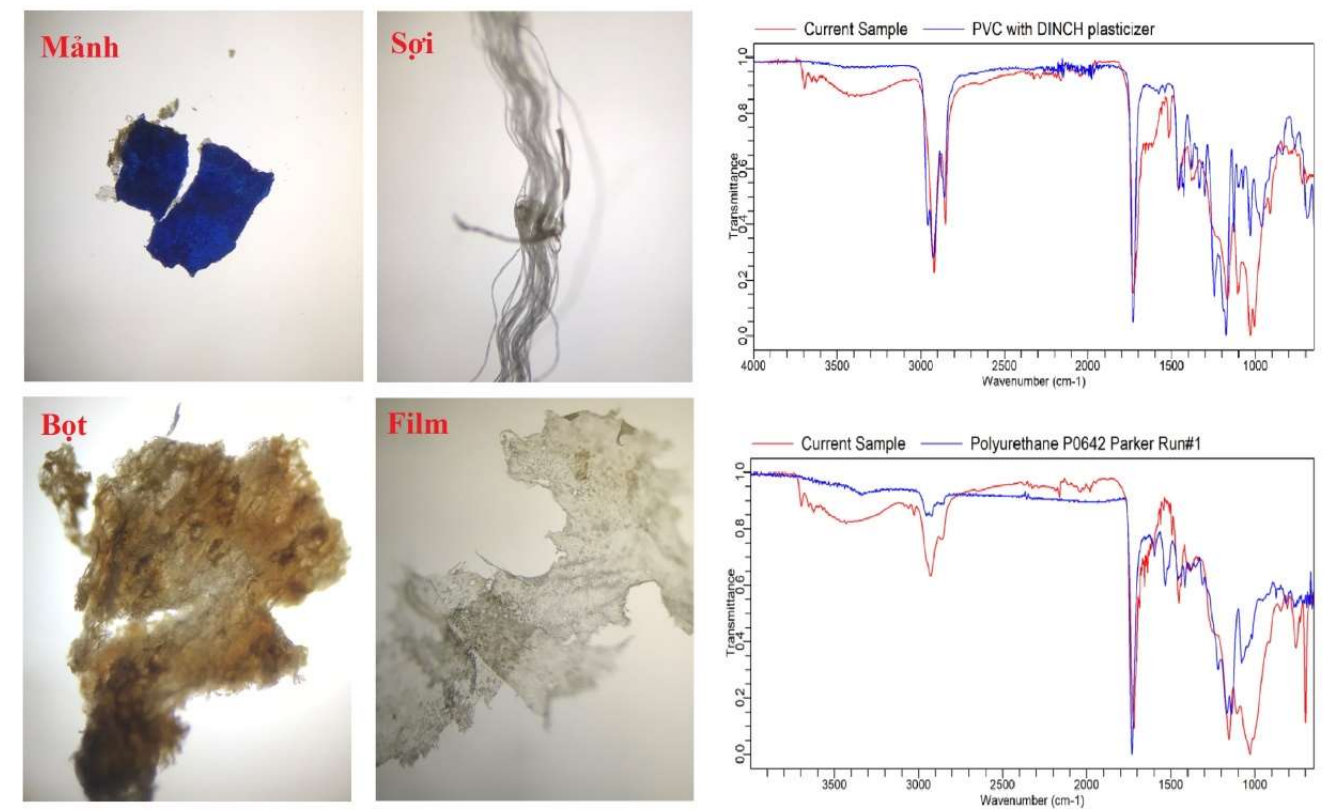

Hình 5. Hình dạng và phân tích FT-IR định danh vi nhựa trong than bùn.

\section{Kết luận}

Kết quả nghiên cứu chỉ ra mức độ nhiễm bẩn vi nhựa ở xã Thuận Nghĩa Hòa, Thạnh Hóa, Long An có hàm lượng dao động trong khoảng giá trị 0-360,0 hạt $/ \mathrm{kg}$. Quá trình phân tích và định danh, thành phần vi nhựa có màu sắc nước biển và trắng chiếm đa số với thành phần tương ứng $52,9 \%$. Liên quan đến kích thước các hạt vi nhựa được tìm thấy trong trầm tích than bùn tồn tại các khoảng giá trị $0,3-0,5 \mathrm{~mm} ; 0,5-1,0 \mathrm{~mm} ; 1,0-3,0 \mathrm{~mm}$; và $3,0-5,0$ $\mathrm{mm}$. Vi nhựa có kích thước tương đối lớn, hình dạng chủ yếu là hình dạng mảnh $(69,6 \%)$, bọt $(17,4 \%)$, sợi $(8,7 \%)$ và dạng film $(4,3 \%)$, chúng thường có màu trắng, nước biển, xanh. Về chủng loại thành phần vi nhựa được phát hiện gồm các loại như $\mathrm{PP}, \mathrm{PE}, \mathrm{PVC}$ và các dạng sợi polyester. Nghiên cứu đã xác định hàm lượng và bước đầu tìm ra một số nguyên nhân gây ra sự nhiễm bẩn. Từ đó cho thấy quá trình nhiễm bẩn rác thải nhựa, đặc biệt ở các vùng sinh thái quan trọng như than bùn là vấn đề môi trường cần được quan tâm cấp bách.

Đóng góp của các tác giả: Xây dựng ý tưởng nghiên cứu: N.M.K., N.T.Q.H.; Phương pháp nghiên cứu: N.M.K., B.Q.D., N.C.M.; Phân tích, đánh giá kết quả: N.M.K., N.T.Q.H., B.Q.D., N.C.M.; Viết bản thảo bài báo: N.M.K.; Chỉnh sửa bài báo: N.T.Q.H., N.M.K., B.Q.D.

Lời cảm ơn: Nghiên cứu này được thực hiện bởi sự tài trợ của WRI Indonesia và Trường Đại học Nông Lâm TP. Hồ Chí Minh. Nhóm tác giả chân thành cảm ơn xã Nghĩa Thuận 
Hòa, huyện Thạnh Hóa, tỉnh Long An đã hỗ trợ trong quá trình khảo sát, thực hiện nghiên cứu này. Đồng thời, tập thể các tác giả xin chân thành cảm ơn các phản biện, đồng nghiệp đã góp ý hoàn thiện bài viết.

Lời cam đoan: Tập thể tác giả cam đoan bài báo này là công trình nghiên cứu của tập thể tác giả, chưa được công bố ở đâu, không được sao chép từ những nghiên cứu trước đây; không có sự tranh chấp lợi ích trong nhóm tác giả.

\section{Tài liệu tham khảo}

1. PlasticEurope. Plastics-the Facts 2017. An analysis of European latest plastics production, demand and waste data. The Association of Plastics Manufacturers in Europe, Brussels, 2018.

2. Andrady, A.L. Microplastics in the marine environment. Mar. Pollut. Bull. 2011, 62, 1596-1605.

3. Novotna, K.; Cermakova, L.; Pivokonska, L.; Cajthaml, T.; Pivokonsky, M. Microplastics in drinking water treatment-Current knowledge and research needs. Sci. Total Environ. 2019, 667, 730-740.

4. Yao, P.; Zhou, B.; Lu, Y.; Yin, Y.; Zong, Y.; Chen, M.-T.; O'Donnell, Z. A review of microplastics in sediments: Spatial and temporal occurrences, biological effects, and analytic methods. Quat. Int. 2019, 519, 274-281.

5. Phuong, N.N.; Poirier, L.; Lagarde, F.; Kamari, A.; Zalouk-Vergnoux, A. Microplastic abundance and characteristics in French Atlantic coastal sediments using a new extraction method. Environ. Pollut. 2018, 243, 228-237.

6. Alomar, C.; Estarellas, F.; Deudero, S. Microplastics in the Mediterranean Sea: Deposition in coastal shallow sediments, spatial variation and preferential grain size. Mar. Environ. Res. 2016, 115, 1-10.

7. Wu, C.; Zhang, K.; Xiong, X. Microplastic pollution in inland waters focusing on Asia. In Freshwater microplastics. The Handbook of Environmental Chemistry, Springer, Cham. 2018, 58, 85-99.

8. Lahens, L.; Strady, E.; Kieu-Le, T.C.; Dris, R.; Boukerma, K.; Rinnert, E.; Gasperi, J.; Tassin, B. Macroplastic and microplastic contamination assessment of a tropical river (Saigon River, Vietnam) transversed by a developing megacity. Environ. Pollut. 2018, 236, 661-671.

9. Jambeck, J.R.; Geyer, R.; Wilcox, C.; Siegler, T.R.; Perryman, M.; Andrady, A.; Narayan, R.; Law, K.L. Plastic waste inputs from land into the ocean. Science 2015, 347, 768-771.

10. Nguyen, Q.A.T.; Nguyen, H.N.Y.; Strady, E.; Nguyen, Q.T.; Trinh-Dang, M. Characteristics of microplastics in shoreline sediments from a tropical and urbanized beach (Da Nang, Vietnam). Mar. Pollut. Bull. 2020, 161, 111768.

11. Phú, H.; Hân, H.T.N.; Thảo, N.L.N.; Đông, Đ.V.; Hân, T.G. Nghiên cứu mức độ ô nhiễm vi nhựa trong nước và trầm tích sông Sài Gòn-Đồng Nai. Tạp chi Khi tương Thủy văn 2021, 731, 69-81.

12. Dũng, L.V.; Dực, T.H.; Hà, N.T.H.; Tùng, N.D.; Tuệ, N.T.; Hiếu, P.V.; Định, N.Q.; Nhuận, M.T. Nghiên cứu phương pháp xác định hạt vi nhựa trong môi trường trầm tích bãi triều ven biển, áp dụng thử nghiệm tại xã Đa Lộc, huyện Hậu Lộc, tỉnh Thanh Hóa. Tạp chí Khí tượng Thủy văn 2020, 715, 1-12.

13. Phuong, N.N.; Duong, T.T.; Pham, Q.T.; Le, Q.T.P.; Amiard, F. Contamination of microplastics in bivalve: First evaluation in Vietnam. VJES 2019, 41(3), 252-258.

14. Hien, T.T.; Nhon, N.T.T.; Thu, V.T.M.; Nguyen, N.T. The Distribution of Microplastics in Beach Sand in Tien Giang Province and Vung Tau City, Vietnam. J. Eng. Technol. Sci. 2020, 52, 208.

15. Karami, A.; Golieskardi, A.; Choo, C.K.; Larat, V.; Karbalaei, S.; Salamatinia, B. Microplastic and mesoplastic contamination in canned sardines and sprats. Sci. Total Environ. 2018, 612, 1380-1386. 
16. Truong, T.N.S.; Strady, E.; Kieu-Le, T.C.; Tran, Q.V.; Le, T.M.T.; Thuong, Q.T. Microplastic in atmospheric fallouts of a developing Southeast Asian megacity under tropical climate. Chemosphere 2021, 272, 129874.

17. Gore, A.J.P. Ecosystems of the World-Mires: Swamps, Bog, Fen, and Moor: New York, NY: Elsevier, 1983.

18. Wright, S.L.; Kelly, F.J. Plastic and human health: a micro issue? Environ. Sci. Technol. 2017, 51, 6634-6647.

19. Phương, T.; Lưu, Q.T.; Lê, T.H. Địa chí Long An: Nhà xuất bản Long An, 1989.

20. SEApeatProject. Distribution and Status of Peatlands in Vietnam: Sustainable Management of Peatland Forests in Southeast Asia, 2010.

21. Bullock, C.H.; Collier, M.J.; Convery, F. Peatlands, their economic value and priorities for their future management-The example of Ireland. Land Use Policy 2012, 29, 921-928.

22. Glenk, K.; Martin-Ortega, J. The economics of peatland restoration. J. Environ. Econ. Manage. 2018, 7, 345-362.

23. Masura, J.; Baker, J.; Foster, G.; Arthur, C. Laboratory Methods for the Analysis of Microplastics in the Marine Environment: Recommendations for quantifying synthetic particles in waters and sediments: The NOAA Marine Debris Program 2015.

24. Anik, A.H.; Hossain, S.; Alam, M.; Sultan, M.B.; Hasnine, M.T.; Rahman, M.M. Microplastics Pollution: A Comprehensive Review on the Sources, Fates, Effects, and Potential Remediation. Environ. Nanotechnol. Monit. Manag. 2021, 16, 100530 .

25. Bui, X.T.; Nguyen, P.T.; Nguyen, V.T.; Dao, T.S.; Nguyen, P.D. Microplastics pollution in wastewater: Characteristics, occurrence and removal technologies. Environ. Technol. Innov. 2020, 19, 101013.

26. Tian, L.; Jinjin, C.; Ji, R.; Ma, Y.; Yu, X. Microplastics in agricultural soils: Sources, effects and their fate. Curr. Opin. Environ. Sci. Health. 2021, 100311.

27. Kiessling, T.; Knickmeier, K.; Kruse, K.; Gatta-Rosemary, M.; Nauendorf, A.; Brennecke, D.; Thiel, L.; Wichels, A.; Parchmann, I.; Körtzinger, A., et al. Schoolchildren discover hotspots of floating plastic litter in rivers using a largescale collaborative approach. Sci. Total Environ. 2021, 789, 147849.

28. Vianello, A.; Boldrin, A.; Guerriero, P.; Moschino, V.; Rella, R.; Sturaro, A.; Da Ros, L. Microplastic particles in sediments of Lagoon of Venice, Italy: First observations on occurrence, spatial patterns and identification. Estuar. Coast. Shelf Sci. 2013, 130, 54-61.

29. Mani, T.; Hauk, A.; Walter, U.; Burkhardt-Holm, P. Microplastics profile along the Rhine River. Sci. Rep. 2015, 5, 17988.

30. Strady, E.; Dang, T.H.; Dao, T.D.; Dinh, H.N.; Do, T.T.D.; Duong, T.N.; Duong, T.T.; Hoang, D.A.; Kieu-Le, T.C.; Le, T.P.Q. Baseline assessment of microplastic concentrations in marine and freshwater environments of a developing Southeast Asian country, Viet Nam. Mar. Pollut. Bull. 2021, 162, 111870.

31. Tibbetts, J.; Krause, S.; Lynch, I.; Sambrook Smith, G.H. Abundance, distribution, and drivers of microplastic contamination in urban river environments. Water 2018, $10,1597$.

32. Mistri, M.; Infantini, V.; Scoponi, M.; Granata, T.; Moruzzi, L.; Massara, F.; De Donati, M.; Munari, C. Microplastics in marine sediments in the area of Pianosa Island (Central Adriatic Sea). Rend. Lincei Sci. Fis. Nat. 2018, 29, 805-809.

33. Lindeque, P.K.; Cole, M.; Coppock, R.L.; Lewis, C.N.; Miller, R.Z.; Watts, A.J.; Wilson-McNeal, A.; Wright, S.L.; Galloway, T.S. Are we underestimating microplastic abundance in the marine environment? A comparison of microplastic capture with nets of different mesh-size. Environ. Pollut. 2020, 265, 114721. 
34. Isobe, A.; Azuma, T.; Cordova, M.R.; Cózar, A.; Galgani, F.; Hagita, R.; Kanhai, L.D.; Imai, K.; Iwasaki, S.; Kako, S.I. A multilevel dataset of microplastic abundance in the world's upper ocean and the Laurentian Great Lakes. Microplastics Nanoplastics 2021, 1, 1-14.

\title{
Characteristics and accumulation of microplastics in peatland of Thuan Nghia Hoa commune, Thanh Hoa district, Long An province
}

\author{
Nguyen Minh Ky ${ }^{1,2 *}$, Nguyen Tri Quang Hung ${ }^{2}$, Nguyen Cong Manh², Bach Quang \\ Dung $^{3}$ \\ ${ }^{1}$ Faculty of Environment and Natural Resources, Nong Lam University - Ho Chi Minh \\ City; quanghungmt@hcmuaf.edu.vn \\ ${ }^{2}$ Department of Environment and Natural Resources, Nong Lam University of Ho Chi \\ Minh City - Gia Lai Branch; nmky@hcmuaf.edu.vn \\ ${ }^{3}$ Room 1703, Apartment 25, Tan Mai Street, Tan Mai Ward, Hoang Mai District, Ha Noi \\ City; dungmmu05@gmail.com
}

\begin{abstract}
The purpose of the study was to evaluate the characteristics and accumulation of microplastics in peatland, a case study in Long An province. The study conducted field surveys and selected representative sampling sites at ten different peatland areas in Thuan Nghia Hoa commune, Thanh Hoa district, Long An province. Samples are processed to determine the content, particle size, colour, shape and identification of their microplastics. The levels of microplastic contamination showed that the average value fluctuated from 0 to 360.0 items $/ \mathrm{kg}$ in the peatland in Long An. Microplastics had a large size, their shape was mainly in the form of the fragment (69.6\%), foam $(17.4 \%)$, fiber $(8.7 \%)$ and film $(4.3 \%)$, and they were white, aqua, blue. Regarding the types of microplastic components detected include polypropylene (PP), polyethylene (PE), polyvinyl chloride (PVC) and polyester fibers; in which, PVC mainly accounted for $70.6 \%$. The findings also demonstrated the spatial distribution of microplastics and indicated signals of humanrelated impacts on the status quo of peatland quality. Therefore, in the long term, it is necessary to propose appropriate solutions to effectively manage and protect natural resources (i.e., peatland) against the pressures of socio-economic development.
\end{abstract}

Keywords: Microplastics; Contamination; PVC; Peatland; Polypropylene; Accumulation. 